\title{
Preface. Conditional: Conceptual and Historical Analysis
}

\author{
Fabien Schang \\ Universida de Estadual de Maringá, \\ Brazil \\ e-mail: schangfabien@gmail.com
}

\begin{abstract}
The logic of conditional is developed hereby in a series of papers, contributing to a historical and critical analysis of what the logical constant is expected to mean.

Keywords: assertion, commitment, conditional, implication, connection, Frege-Geach argument, relevance.
\end{abstract}

Conditional is certainly one of the most controversial logical constants. Even a radical logical pluralist will concede this point, despite the relative meaning of the so-called "syntecategorematics" in natural language, there are not so many writings about the ambiguity of conjunction or disjunction than about conditional. This is mainly due to the famous ambiguity between object language and metalanguage, famously noted by Willard van Orman Quine and focusing on the close similarity between conditional and deduction. The same confusion may be mentioned about the constant of negation, all the more that the problematic meaning turns out to be also related in the characterization of conditional. Admittedly, it is not an easy task to clarify the debate evolving around what is currently dubbed as either "conditional" or "implication". A number of conditionals occurred in the contemporary history of logic: material strict, intuitionistic, relevant, linear, and so on. Whether classical or non-classical, a common difficulty comes from the nature of the "nexus" between the antecedent and the consequent. Should there be a causal, temporal, or merely casual relation between them?

The present issue does not want to give an exhaustive survey of the literature having to do with conditional. However, some of the most renown problems of logic are discussed: the paradoxes of material implication, to the effect that a conditional is true whenever its antecedent is false; Russell's "Embedding Problem" (or the Frege-Geach Problem), which deals with the status of assertion and the troublesome role of antecedent in conditional statements; the problem of pure implication, i.e., how to find a proper characterization of conditional which makes no use of other logical constants in its definition and makes it differ from the other ones.

In order to disentangle such a thorough discussion around conditional, the present issue intends to bring its own contribution to the debate around what conditional means. For this purpose, this special issue proposes a twofold reading of the logical constants in its conceptual and historical 
aspects. Two famous inference rules, Modus Ponens and the Deduction Theorem, cannot be neglected in such an enterprise.

In "The Semantics and Pragmatics of Conditional in al-Farabi and Avicenna", Saloua Chatti proposes a comparative analysis between the logical analyses of conditional by al-Farabi and Avicenna. Beyond a various taxonomy in their respective writings, it clearly appears that a number of common features emerge from the account of conditional in such Arabic medieval commentaries. Thus temporal, causal, and strictly logical connections between antecedent and consequent are clearly mentioned therein, thus showing that what still prevails in the modern literature around conditional was already emphasized in both Aristotelian and post-Stoician commentators.

In "Implications and Limits of Sequences", Alexandre Costa-Leite and Edelcio Souza challenge the existence of essential properties for conditional in natural language. Accordingly, the historical aspects of conditional are kept aside from this paper. Rather, the authors favor a normative approach over the descriptive one and lead to an original definition of conditional in mathematical terms of limits in a finite sequence of sentences. The latter is promoted as a more promising account than the truth-functional, lattice-theoretical, or structural one (by Arnold Koslow).

In "Assertions and Conditionals: A Historical and Pragmatic Stance", Daniele Chiffi and Alfredo Di Giorgio combine a more recent historical approach with a modern formal language of pragmatics. Thanks to a thorough survey of medieval philosophers and logicians including Abelardus, Ockam, or Bricot, the authors want to show that a serious "assertion candidate" requires an introduction of two additional concepts, viz. assertion and judgment. The illocutionary import of conditional thus leads to a formal logic, Logic for Pragmatics, where the distinction between radicals (propositional contents with no assertive force) and sentences (asserted propositions) helps to redefine conditional in close connection with intuitionistic and modal logics.

In "Conditionals in Interaction", James Trafford also advocates the assertoric import of conditional, by attempting to clarify the distinction between the assertion of a conditional and a conditional assertion. After reminding the objective reading of assertion within the antirealist literature, the author defends a social view of assertion within a social community of speakers: in the line of Brandom's view of logic as a "game of giving and asking for reasons", conditional should better be understood as a whole hypothetical move rather than a categorical commitment performed by a speaker upon the antecedent.

In "The Football of Logic", Fabien Schang follows Trafford's dynamic characterization of conditional in a game-theoretical description of proof. More than that, the social aspect of logic is emphasized by an analogy between logic and football. Through the Tarskian framework of logic as truth-preservation, Schang argues that the players of the two kinds of game equally purport to preserve something whilst aiming at a goal against another player. A "strong" version of conditional finally results from a structured interpretation of truth-values, both erasing the paradoxes of material implication and adapting the dialogical question-answer game into a four-valued algebraic logic. 